Disclosure of Interests: Melissa Domaille: None declared, Paul Whybrow: None declared, Elizabeth Carver-Richardson: None declared, Emma Dures Grant/research support from: Has previously received an independent learning grant from Pfizer, however the work has been completed and the grant has been closed., Rosemary Greenwood: None declared, Pamela Richards: None declared, Joanna Robson: None declared, Robert Stellinga: None declared, Fiona Cramp: None declared

DOI: 10.1136/annrheumdis-2019-eular.965

\section{FRI0708-HPR PROGNOSTIC FACTORS ASSOCIATED WITH AN EARLY RESPONSE TO PHYSIOTHERAPY TREATMENT IN PATIENTS WITH CHRONIC NONSPECIFIC NECK PAIN: AN EXPLORATORY PROGNOSTIC MODEL}

Lucia Domingues $^{1,2}$, Eduardo B. Cruz ${ }^{3}$, Fernando Pimentel Dos Santos ${ }^{1,4}$, Jaime Branco ${ }^{1,4} .{ }^{1}$ CEDOC-Chronic Diseases Research Center-Nova Medical School/Faculdade de Ciências Médicas, Rheumatic Diseases, Lisbon, Portugal; ${ }^{2}$ Centro de Medicina e Reabilitação de Alcoitão, Lisbon, Portugal; ${ }^{3}$ Escola Superior de Saúde - Instituto Politécnico de Setúbal, Physiotherapy Department, Setúbal, Portugal; ${ }^{4} \mathrm{CH}$ LO/Hospital Egas Moniz, Rheumatology Department, Lisbon, Portugal

Background: Chronic nonspecific neck pain (CNP) is a common health problem worldwide. Previous studies identified sociodemographic and clinical factors associated with successful outcomes in patients at discharge of physiotherapy treatment. However, the prognostic factors associated with an early response to physiotherapy treatment in patients with CNP are unclear. This knowledge may allow to identify a profile of patients with higher odds of improvement at the beginning of treatment, supporting clinical decision-making considering benefits versus non-benefits at shortterm.

Objectives: This study aimed to identify prognostic factors associated with an early successful response to Physiotherapy treatment in patients with CNP. The successful response was defined as a reduction on disability of $\geq 30 \%$ after 3 -weeks of physiotherapy treatment.

Methods: A prospective cohort study was conducted on 52 patients with CNP lasting $\geq 3$ months, undergoing a physiotherapy treatment programme of mobilisation and exercise (coordination, strength, endurance). Patients were assessed at baseline, and then 3 -weeks later. Participants were categorised as having a successful outcome if they scored a difference in their disability above the Minimal Clinical Important Difference (MCID) of the Neck Disability Index (NDI). Logistic regression analysis (backward stepwise conditional method) was used to identify the associations between baseline prognostic factors and outcome. Socio-demographic and clinical characteristics of CNP were included as potential prognostic factors

Results: A total of 51 participants completed the intervention. At 3-weeks post-treatment, $75 \%(38 / 51)$ of the participants achieved a successful response to physiotherapy treatment. In the final multivariate model (Omnibus Tests $\mathrm{p}<0.001$ ), an early successful response to Physiotherapy treatment was significantly associated with the disability score (OR 1.16 $\mathrm{Cl} 95 \%$ 1.02-1.32), and pain intensity (OR $1.81-\mathrm{Cl} 95 \% 1.03-3.20$ ) at the baseline. This model improves the classification ability from 74.5 to $86.3 \%$, explaining $50.6 \%$ of the outcome, with good predictive ability of sensibility (94.5\%) and modest specificity (61.5\%). The area under the ROC curve for disability score $(0.8 ; 95 \% \mathrm{Cl}$ : $0.6-0.9)$ and pain intensity $(0.7 ; 95 \% \mathrm{Cl}: 0.5-0.9)$ indicated good and acceptable discriminatory ability, respectively. After 3 -weeks of mobilisation and exercise, the patients with scores $\geq 12$ on NDI and $\geq 7$ on Numeric Pain Rating Scale at baseline have increased odds of achieving an early response to treatment in the presence of both variables ( $+\mathrm{LR}=1.7195 \% \mathrm{Cl}$ : $0.84-3.50)$ or one variable (+LR=1.45 95\% Cl: 0.69-3.04)

Conclusion: This study suggests that patients with medium to high levels of disability and high levels of pain at the baseline, treated with a physiotherapy programme of mobilisation and exercise, are more likely to experience an early reduction on their disability score. References:

Disclosure of Interests: Lucia Domingues: None declared, Eduardo B. Cruz: None declared, Fernando Pimentel dos Santos Grant/research support from: From Abbvie and Novartis, Speakers bureau: Abbvie, Novartis, Pfizer, Biogen, Jaime Branco: None declared

DOI: 10.1136/annrheumdis-2019-eular.4377
FRI0709-HPR EFFECTS OF LAND- AND WATER-BASED EXERCISE INTERVENTIONS ON PAIN IN PEOPLE WITH FIBROMYALGIA: A PRELIMINARY REPORT FROM THE AL-ÁNDALUS RANDOMISED CONTROLLED TRIAL

Fernando Estevez-Lopez ${ }^{1,2}$, Inmaculada C. Alvarez-Gallardo ${ }^{3}$, Víctor SeguraJiménez ${ }^{3}$, Milkana Borges Cosic ${ }^{1}$, Pedro Acosta-Manzano ${ }^{1}$, Blanca Gavilán Carrera ${ }^{1}$, Ana Carbonell-Baeza ${ }^{3}$, Manuel Delgado-Fernández ${ }^{1}$, Virginia A. Aparicio ${ }^{1}{ }^{1}$ University of Granada, Granada, Spain; ${ }^{2}$ Ulster University, Belfast, United Kingdom; ${ }^{3}$ University of Cádiz, Cádiz, Spain

Background: Non-pharmacological approaches are the mainstay of treatment in fibromyalgia. The current recommendations of the European League Against Rheumatism (EULAR) for the management of fibromyalgia highlight that exercise is the only therapy with a 'strong' evidence [1]. Exercise has been typically implemented on either land- or water-based settings. However, it is unclear whether to perform exercise in different settings has different effects on pain; this knowledge might help to maximise the beneficial effects that exercise has in fibromyalgia [2]

Objectives: To compare the effects of two exercise interventions (landand water-based training) on pain in people with fibromyalgia.

Methods: From 272 initially randomized, a total of 151 participants (50.6 \pm 7.6 years old, $98 \%$ women) completed all the assessments and attended to at least $70 \%$ of the programme; 48,42 and 61 participants pertained to the land-based exercise, water-based exercise, and usual care (control) groups, respectively. The intervention groups trained 3 nonconsecutive days/week (45-60 minutes per session) for 24 weeks. Each session included aerobic exercises, muscular strengthening and stretching for all the major muscle groups. Pain was measured by the 0-100 mm visual analogue scale (VAS) from the Fibromyalgia Impact Questionnaire (FIQ). Catastrophizing and self-efficacy pain-related cognitions were assessed by the Pain Catastrophizing Scale (PCS total score) and pain management subscale (PSE) of the Chronic Pain Self-efficacy Scale (CPSS), respectively. We calculated an algometer score based on the sum of pain thresholds $(\mathrm{kg} / \mathrm{cm} 2)$ of the 18 tender pints according to the 1990 American College of Rheumatology fibromyalgia diagnostic criteria. Participants were evaluated at baseline (pre-test), at the end of the 24week intervention (post-test) and after a 12-week detraining period (retest).

The groups were comparable in sociodemographic and clinical characteris tics; they only differ on age, which was included as a covariate along with baseline levels of pain.

Results: Adjusting for Bonferroni, most of the between-group comparisons of pain changes over time were not significant. As exceptions, in comparison to the control group, participants in the land-based exercise group lowered catastrophizing and improved algometer score at the post-tests mean difference $(95 \%$ interval confidence) $[\mathrm{MD}(95 \% \mathrm{Cl})]=-4.0(-7.5$ to $0.5)$ and 6.2 (2.0 to 10.5), respectively. These differences became nonsignificant at the re-test.

Conclusion: These preliminary results suggest that a 24-week land-based exercise intervention had beneficial effects by reducing pain catastrophizing and increasing algometer score in people with fibromyalgia. However, these benefits were unsustained after the detraining period. In compassion to the control group, a water-based exercise intervention did not show any effect on pain. Although our finding suggest that a land-based exercise intervention may have short-term beneficial effects on pain, these findings must be considered as preliminary until more robust analyses are performed.

\section{REFERENCES:}

[1] Macfarlane GJ, et al. Ann Rheum Dis 2017;76:318-28. Doi: :10.1136/annrheumdis-2016-209724

[2] Carbonell-Baeza A, et al. BMC Musculoskelet Disord 2012;13:18. Doi:10.1186/1471-2474-13-18

Acknowledgement: This study was supported by the Spanish Ministry of Economy and Competitiveness (I+D+i DEP2010-15639; I+D+I DEP201340908-R; BES-2014-067612) and the Spanish Ministry of Education (FPU14/02518; FPU 15/00002)

Disclosure of Interests: None declared

DOI: 10.1136/annrheumdis-2019-eular.7337 